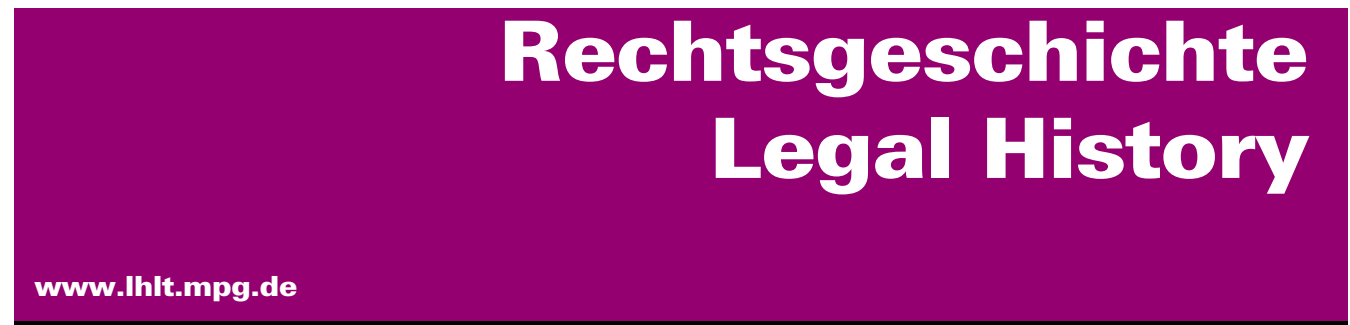

http://www.rg-rechtsgeschichte.de/rg29

$\operatorname{Rg} 292021 \quad 335-337$

Zitiervorschlag: Rechtsgeschichte - Legal History Rg 29 (2021)

http://dx.doi.org/10.12946/rg29/335-337

\title{
Alexander Kästner*
}

\section{Urteilen über Suizid als soziale Praxis}

[Judging Suicide as a Social Practice]

* TU Dresden, Institut für Geschichte, alexander.kaestner@tu-dresden.de

Dieser Beitrag steht unter einer Creative Commons Attribution 4.0 International License 
Reichsadel verbleibt. Betont wird die Bedeutung des Richteramtes für das symbolische, soziale und politische Kapital mindermächtiger Adelsfamilien und die Nutzung als Ressource, um im Kontext von adligen und kaiserlichen Klientelnetzwerken Einfluss auf die Reichsjustiz zu nehmen. Die für die reichskammergerichtliche Praxis untersuchten Bereiche und Konflikte belegen das Spannungsverhältnis von justizieller Autonomie und Ausdifferenzierung des Rechts zu den Normen der vormodernen Adelsgesellschaft und einem traditionalen Verständnis des Richteramtes als (symbolisch-zeremonielle) Repräsentanz des Kaisers als dem höchsten Gerichtsherren. Zwischen kaiserlichen Interessen, Klientelnetzwerken und Ausübung des höchsten richterlichen Amtes blieb die Rolle des Kammerrichters im Reichskammergericht prekär und bedurfte beständiger Absicherung durch Zeremoniell und symbolisches Kapital.
Eine tiefergehende Analyse, inwiefern sich diese für das Reichskammergericht und das Amt des Kammerrichters treffend beobachteten Konstellationen wandelten, gibt von Loewenich allerdings nicht. Weder erfolgt eine Diskussion und Einordnung der Ergebnisse in die Forschung noch nutzt die Autorin das vorhandene Potential eines Vergleichs mit Reichshofrat und Reichstag oder der allgemeinen Entwicklung von Richteramt und Justiz in der Frühen Neuzeit. Insofern beschränkt sich der Erkenntnisfortschritt der Arbeit auf valide und auch rechtshistorisch interessante empirische Beobachtungen für Reichskammergericht und Kammerrichter, weitergehende und insbesondere rechtshistorische Deutungs- und Vergleichsperspektiven bleiben freilich den Leserinnen und Lesern überlassen.

\section{Alexander Kästner Urteilen über Suizid als soziale Praxis*}

Das vorliegende Buch ist die stark gekürzte Version einer Dissertation, die 2015 an der Universität Tampere eingereicht wurde. Der ursprüngliche Titel lautet "Suicide in Seventeenth-Century Sweden. The Crime and Legal Praxis in the Lower Courts«. Er verweist präziser auf den zeitlichen Zuschnitt der Studie und auf den maßgeblich zugrundeliegenden Quellenbestand an Gerichtsakten schwedischer Stadt- und Landgerichte. Für die Überarbeitung wurden wesentliche Teile der ursprünglichen Darstellung gestrafft, was insbesondere den Details und der Tiefe einzelner Forschungsdiskussionen zum Nachteil gereicht, soweit ich das überblicke - die ursprüngliche Dissertation wurde mir von der Verfasserin freundlicherweise zur Verfügung gestellt. »Suicide, Law, and
Community in Early Modern Sweden « ist ein Buch, das sich an ein breiteres akademisches Publikum auch jenseits der Historischen Suizidforschung richtet und vor allem übergreifende Befunde und ausgewählte Fälle präsentiert. Dies sollte vor der Lektüre klar sein, um zu verstehen, weshalb insbesondere die Einleitung und das Fazit eher allgemein gehalten sind und das Fazit auch keine weiterführenden Forschungsperspektiven aufzeigt. Als eher unglücklich muss die verlegerische Entscheidung bezeichnet werden, jedem Kapitel ein gesondertes Quellen- und Literaturverzeichnis folgen zu lassen. Das geht zu Lasten des Umfangs und erweckt auch den falschen Eindruck, es würde sich bei den Kapiteln um jeweils eigenständige Aufsätze handeln.

\footnotetext{
RIIKKA MIETTINEN, Suicide, Law, and Community in Early Modern Sweden (World Histories of Crime, Culture and Violence), Basingstoke: Palgrave Macmillan 2019, XII + 346 S., ISBN 978-3-030-11844-0
} 
Die Studie ist empirisch stark aus dem historischen Quellenmaterial heraus entwickelt und betrachtet ca. 200 Fälle - die angegebene Anzahl variiert zwischen den Kapiteln aufgrund der Überlieferung und Informationsdichte $\mathrm{zu}$ einzelnen Fällen auf den unterschiedlichen Gerichtsebenen. Eine Karte (14) verdeutlicht die beeindruckende räumliche Dimension der Studie, die neben den Territorien des heutigen Zentral- und Nordschwedens auch die finnischen Gebiete Schwedens während der Großmachtzeit einschließt sowie die mit Russland umkämpften karelischen Territorien um Wyborg und Kexholm (Priosersk). Dieser Umstand ist strafrechtshistorisch spannend, da in den angrenzenden Gebieten der Nowgoroder Rus bzw. im Großfürstentum Moskau Selbsttötungen bis ins 18. Jahrhundert nicht als Kriminaldelikt galten, während in Zentralschweden bzw. in den Territorien der Kalmarer Union vorsätzliche Selbsttötungen wie in Europa sonst üblich als peccatum vel crimen atrocissimum postmortal bestraft wurden. Die regionalen Besonderheiten und die geringe administrative Durchdringung der Peripherie wirkten sich u.a. auf die vorgerichtliche Praxis des Umgangs mit Suizidenten aus.

Der große Vorzug von Miettinnens Studie gegenüber anderen Untersuchungen zu Selbsttötungen in Schweden besteht darin, konsequent die Bestandteile der Verfahren im Umgang mit (mutmaßlichen) Selbsttötungen zum Gegenstand einer mikrohistorischen Analyse zu machen und zugleich eine Ebene des interterritorialen Vergleichs unterschiedlicher Rechtsräume einzuziehen (die 2016 abgeschlossene Wiener Dissertation von Evelyne Luef fügt dem einen Vergleich zwischen Schweden und den Habsburger Territorien hinzu und ist online verfügbar). In der Konsequenz hangelt sich die Kapitelgliederung zunächst entlang eines Überblicks über Einstellungen gegenüber Selbsttötungen und den unmittelbaren Reaktionen auf konkrete Suizidfälle nach dem Fund einer verdächtigen Leiche (Kap. 2). Ein Ausgangspunkt sind die über Jahrhunderte hinweg diskutierten schematischen Bestimmungen für Selbsttötungen (Verbrennung der Leiche im Wald oder Schandbegräbnis an abgelegenen Orten) in Kristofers Landslag von 1442. Dessen Geltung blieb umstritten, bis es Karl IX. im Jahr 1608 mit Änderungen nachdrucken ließ. Faktisch markiert damit das Jahr 1608 den Beginn des engeren Untersuchungszeitraums, die Einführung des års lag im Jahr 1734 dessen Ende. Die Konsequenzen der Einführung von Appellationsgerichten (hovrätter) für die großen Gerichtsbezirke Schwedens im Jahr 1614 (Stichworte: Zentralisierung, Verrechtlichung, Professionalisierung) bilden den roten Faden für die weitere Erzählung, die sich auch als ein Beitrag zur Rechtskultur Schwedens im 17. Jahrhundert versteht. Ausgangspunkt ist hier die Beobachtung, dass die örtlichen Gerichte in ihren Entscheidungen eng an den Wortlaut der mittelalterlichen Bestimmungen gebunden waren, die entsprechenden Urteile aber zunehmend einer ausdifferenzierten Urteilspraxis der hovrätter unterworfen und revidiert wurden. Entscheidend für erste Reaktionen auf Selbsttötungen waren mitunter die erheblichen Distanzen für die Gerichte (auf dem Land tagten diese dreimal jährlich) und die geringe Siedlungsdichte - wichtig, wenn man bedenkt, dass eine Laienjury bestehend aus zwölf ortsansässigen Landbesitzern gemeinsam mit einem Richter und im Zusammenspiel mit einer Vielzahl weiterer örtlicher Gerichte zu urteilen hatte. Nicht selten blieben Leichen wochen- oder monatelang am Fundort liegen oder hängen, bevor sich ein Gericht mit dem Fall beschäftigte. Für die östlichen Territorien sind auffällig häufig Berührungen der Leiche oder das Abschneiden von Erhängten nach dem Auffinden überliefert, was auf die erwähnten unterschiedlichen Traditionen verweist. Systematisch unterbelichtet bleibt in der gesamten Studie die Beobachtung, dass die lutherischen Kirchengerichte entgegen der Bestimmungen des Kristofers Landslag seit dem letzten Drittel des 16. Jahrhunderts stille Beisetzungen auf dem Friedhof anordnen konnten; entscheidend wird diese Differenz für Miettinen erst, nachdem die Kirchengerichte 1686 dazu verpflichtet wurden, bei der Behandlung mutmaßlich vorsätzlicher Suizidenten die weltlichen Gerichte einzubeziehen ab diesem Zeitpunkt wird auch die Überlieferung spürbar dichter, was vermuten lässt, dass viele Fälle zuvor durch kirchliche Instanzen geregelt wurden. Die Tatsache, dass es sich bei Suiziden um delicta mixta handelte, wird in der Studie nicht gleichgewichtig behandelt. Der Fokus liegt einseitig auf der Überlieferung der weltlichen Gerichte.

Kapitel 3 nimmt die vorgerichtlichen Untersuchungen und die Kommunikation vor Ort in den Blick, die den weiteren Umgang mit den Leichen von Suizidenten und die späteren Verhandlungen von Suizidfällen vor lokalen Gerichten bestimmten. Erhebliche Herausforderungen waren etwa die gerichtliche Bewertung der Aus- 
sagen von Verwandten der Suizidopfer sowie das Rekrutieren einer hinreichenden Anzahl positiv beleumundeter Zeugen. Da es für Gerichte und Amtsträger ebenfalls bis 1686 keinerlei finanzielle Anreize gab, in Suizidfällen zu ermitteln, kam so manche Untersuchung auch nur widerwillig und schleppend in Gang. Zudem spielte die Güterkonfiskation nach Suiziden in Schweden keine Rolle.

Kapitel 4 bildet sodann das Herzstück der Studie. Hier untersucht Miettinnen die konkreten Verhandlungen während der öffentlichen Gerichtstage vor Ort, das Anhören von Zeugen, die Rolle der Schreiber für das Verfassen von Protokollen und den wachsenden Einfluss der Rechtsprechung der hovrätter. Ergänzend werden daher die Bestände des Svea hovrätt in Stockholm herangezogen, während die Überlieferung des ebenfalls wichtigen Turku hovrätt von erheblichen Verlusten geprägt ist. Die lokalen Gerichte werden als soziale Arenen und Schnittstellen staatlicher und lokaler Akteure konzipiert, in denen verschiedene Wahrheitsansprüche verhandelt wurden. Unter den Sachverständigen spielten nur Pfarrer bzw. Ortsvikare als Experten für ein gutes Leben eine signifikante Rolle, sie waren in $40 \%$ der Fälle involviert. Medizinische Expertise war mangels Personal faktisch abwesend. Für die Appellationsgerichte wurden zunehmend Wille und Intention (hier der Rechtsentwicklung im Alten Reich hinterherhinkend) wichtig, die in den mittelalterlichen Bestimmungen gar nicht vorkamen. Dies trug maßgeblich zu einer vielgestaltigen Urteilspraxis bei.

Kapitel 5 fokussiert sodann die Analyse zielgerichtet auf diese Urteilspraxis, auf die Kategorien- bildungen in den Endurteilen sowie auf deren Einbettung in soziale Kontexte. Die Reputation und Integration eines Suizidenten waren ebenso entscheidend für das Urteil wie die Einschätzung von Frömmigkeit, Lebenswandel und persönlichem Charakter. Die Strafe des Verbrennens kam langsam außer Gebrauch; und man könnte über die Studie hinausgehend die These formulieren, dass sich die schwedische Praxis zunehmend der ambivalenten Normalität und ausdifferenzierten Praxis im Umgang mit Selbsttötungen annäherte, wie sie bspw. für die Territorien des Alten Reichs belegt sind. Das mag auch damit zusammenhängen, dass die Richter vor Ort vor ihrer Berufung schrittweise in ein System der praktischen Ausbildung an den Appellationsgerichten eingebunden wurden, deren Urteilspraxis kannten und daher in eigenen Urteilen antizipierten.

Die Leistung der Studie besteht darin, eine detaillierte und innerhalb der schwedischen Rechtsräume vergleichende Analyse zu allen relevanten Akteuren, Institutionen und Verfahrensweisen von der Ebene der Gemeinden bis hin zu den Hochgerichten für den Umgang mit Selbsttötungen vorgelegt zu haben. Eine solche Studie fehlte bislang für den skandinavischen Raum, und sie ist eine mehr als willkommene Ergänzung für die historische Suizidforschung. Mit dem vorliegenden Buch hat Riikka Miettinen den Grundstein für die künftige Einordnung und Perspektivierung weiterer Lokal- und Einzelfallstudien gelegt, wenngleich für manche Details die Dissertationsschrift unverzichtbar bleibt. 